\title{
Idiopathic Pulmonary Hemosiderosis: An Unexplored Cause of Treatment Refractory Pediatric Iron Deficiency Anemia
}

\author{
Anjuna Reghunath ${ }^{1, \oplus} \quad$ Joyutpal Biswas ${ }^{1} \quad$ Mahesh Kumar Mittal ${ }^{1} \quad$ Reeta Kanaujiya ${ }^{1} \quad$ Geetika Khanna ${ }^{2}$ \\ ${ }^{1}$ Department of Radiodiagnosis, Vardhman Mahavir Medical College \\ and Safdarjung Hospital, New Delhi, India \\ 2Department of Pathology, Vardhman Mahavir Medical College and \\ Safdarjung Hospital, New Delhi, India \\ Address for correspondence Reeta Kanaujiya, MD, Department of \\ Radiodiagnosis, Vardhman Mahavir Medical College and Safdarjung \\ Hospital, New Delhi-110029, India \\ (e-mail: reetakanaujia@gmail.com).
}
Abstract
Keywords
- hemoptysis
- pulmonary hemorrhage
- hemosiderosis

Idiopathic pulmonary hemosiderosis (IPH) is an unusual cause of pediatric iron deficiency anemia (IDA) characterized by alveolar hemorrhage leading to hemosiderin deposition and fibrosis in the lungs. Though the typical triad of presentation is hemoptysis, IDA, and lung opacities on thoracic radiographs, often the sole manifestation of $\mathrm{IPH}$ may be severe IDA in children.

\section{Case Report}

An 8-year-old female child presented to us with complaints of cough and acute exacerbation of dyspnea for 4 months. Her mother recalled that the child had easy fatiguability, poor weight gain, and lethargy since 2 years of age. The child had also found it difficult to actively engage in outdoor games with her peer group due to recurrent episodes of dyspnea. There was no history of hemoptysis, fever, contact with tuberculosis/pets, or exposure to factory smoke. The child had been diagnosed with iron deficiency anemia (IDA) in 2014 based on peripheral smear, blood profile, serum iron studies, and bone marrow biopsy done from another institute. Her hemoglobin at that time was only $4 \mathrm{mg} / \mathrm{dL}$ and over 5 years she had had six episodes of blood transfusion and treatment with oral iron and antihelminthics.

Clinical examination revealed grade III clubbing, right parasternal heave, and loud P2 on auscultation. Her blood examination currently showed normal peripheral smear and no evidence of IDA with red blood cell count of 4.9 million $/ \mathrm{mm}^{3}$, hemoglobin $14 \mathrm{mg} / \mathrm{dL}$, hematocrit $46.3 \%$, mean corpuscular volume (MCV) $94.5 \mathrm{fL}$, mean corpuscular hemoglobin $(\mathrm{MCH}) 30.4 \mathrm{pg}$, MCH concentration (MCHC) $32.2 \%$, serum ferritin $506.46 \mathrm{ng} / \mathrm{mL}$, serum iron $44 \mu \mathrm{g} / \mathrm{dL}$, and total iron-binding capacity $378 \mu \mathrm{g} / \mathrm{dL}$. Sputum was negative for tubercular bacilli; however, her Mantoux test was positive. The serum human immunodeficiency virus screen turned out to be negative.

A routine chest radiograph of the child revealed reticular opacities in bilateral middle and lower zones with patchy opacities in the left lower zone, based on which interstitial lung pathology was suspected ( - Fig. 1). Further, a high-resolution computed tomography (HRCT) was done, which showed diffuse bilateral ground-glass opacity (GGO) with a mild apicobasal gradient ( $\bullet$ Fig. 2A). Few thin-walled small cysts were seen in peribronchovascular distribution (-Fig. 2B) in upper lobes along with crazy paving appearance due to superimposed interlobular septal thickening over areas of GGO in upper and lower lobes ( - Fig. 2C). Peripheral and basilar, confluent nodules, and interlobular septal thickening with mild tractional bronchiectasis were observed in the left lower lobe, suggestive of fibrotic changes ( - Fig. 2D). published online July 28,2021
DOI https://doi.org/

10.1055/s-0041-1734334 ISSN 0971-3026
(C) 2021. Indian Radiological Association.

This is an open access article published by Thieme under the terms of the Creative Commons Attribution-NonDerivative-NonCommercial-License, permitting copying and reproduction so long as the original work is given appropriate credit. Contents may not be used for commercial purposes, or adapted, remixed, transformed or built upon. (https://creativecommons.org/licenses/by-nc-nd/4.0/).

Thieme Medical and Scientific Publishers Private Ltd. A-12, Second Floor, Sector -2, NOIDA -201301, India 


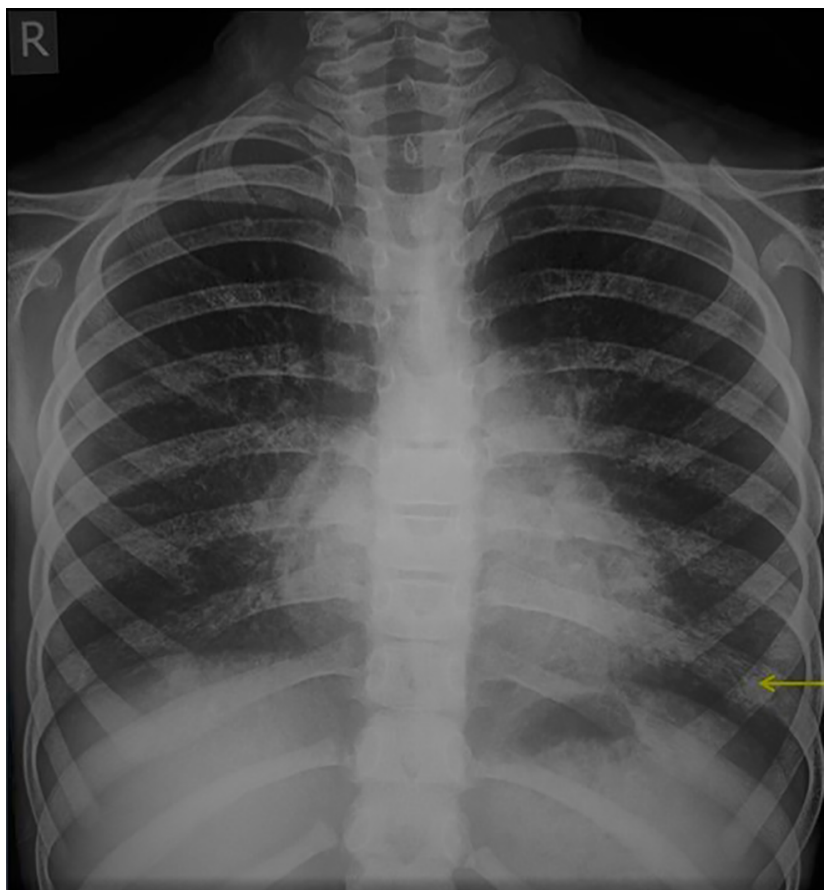

Fig. 1 Chest radiograph of the child showing reticular opacities in bilateral mid and lower lung zones with patchy fibrotic opacity (yellow arrow) in left lower zone.
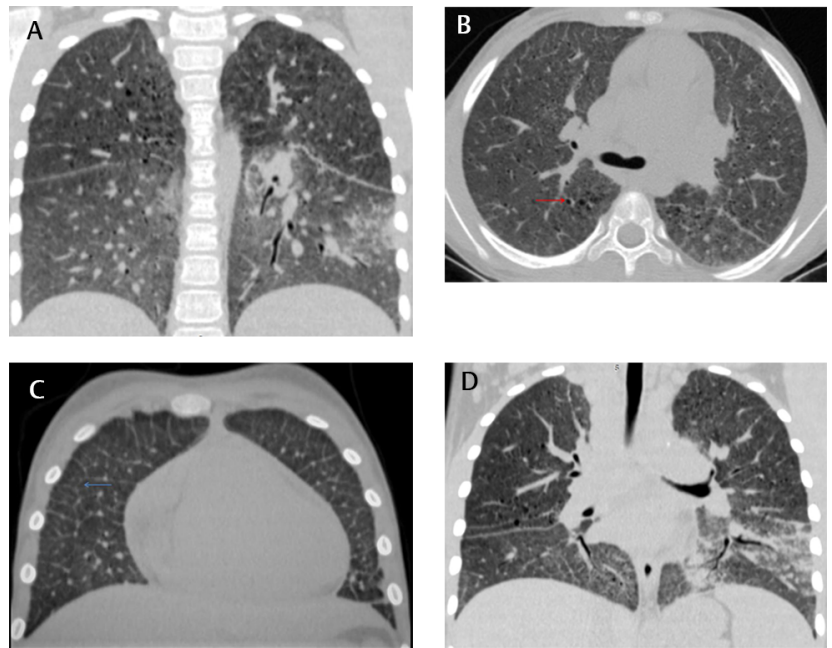

Fig. 2 (A-D) High-resolution computed tomography (CT) of thorax revealing diffuse ground-glass opacity of both lung fields with apicobasal gradient (A), few small cystic lesions in peribronchovascular distribution suggesting honeycombing (red arrow) (B), bilateral interlobular septal thickening superimposed on areas of ground-glass opacity, giving "crazy paving appearance" (blue arrow) (C), and confluent fibrotic nodules in left lower lobe with mild tractional bronchiectasis (D).

HRCT was followed by contrast-enhanced CT (CECT) due to clinical suspicion of pulmonary artery hypertension $(\mathrm{PAH})$ on the mediastinal window of the scan and also to rule out any necrotic lymphadenopathy. CECT depicted PAH ( - Fig. 3) and homogenously enhancing mediastinal lymph nodes.

Based on the imaging findings and history of IDA, the possibility of pulmonary hemosiderosis was considered. A detailed vasculitic work-up including serum antineutrophil

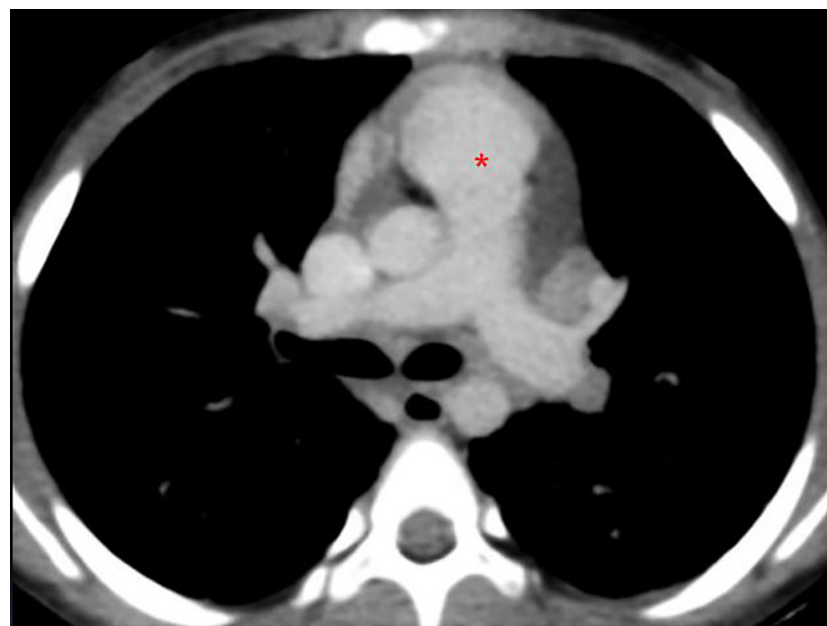

Fig. 3 Contrast-enhanced computed tomography (CT) in mediastinal window demonstrates dilated main pulmonary artery (asterisk).
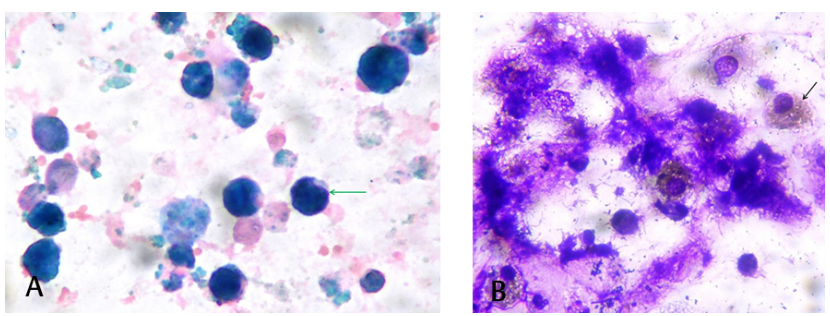

Fig. $4(\mathbf{A}, \mathbf{B})$ Cytopathological examination of bronchoalveolar lavage fluid with Perl's Prussian blue stain revealing presence hemosiderin-laden macrophages (green arrow) (A). On evaluation with May-Grunwald-Giemsa stain, the hemosiderin engulfed by siderophages stain brown (black arrow) (B), confirming the diagnosis of pulmonary hemosiderosis.

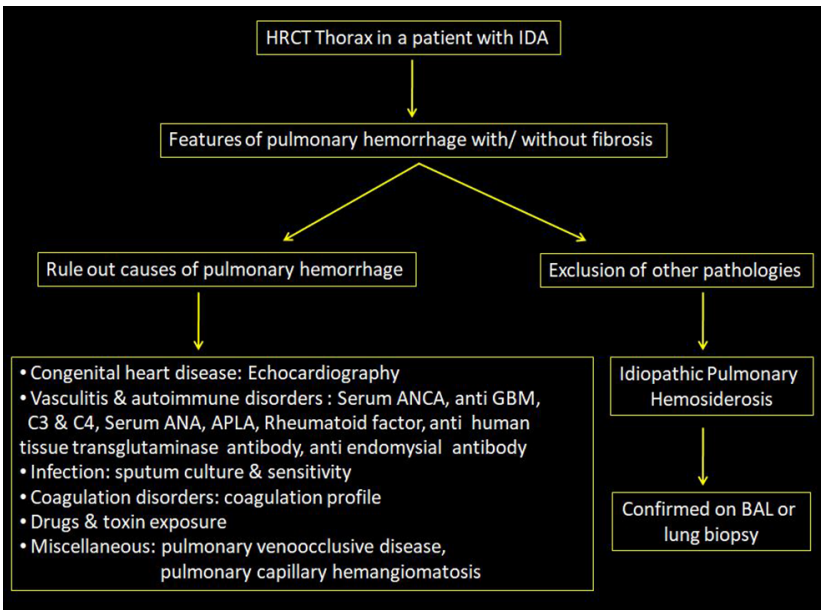

Fig. 5 Algorithm for approach to pulmonary hemosiderosis.

cytoplasmic antibody (ANCA), antinuclear antibody profile, antiphospholipid antibody (immunoglobulin [Ig] M and IgG), antiglomerular basement membrane antibody, anti-human tissue transglutaminase antibody, anti-endomysial antibody (IgA), rheumatoid factor, C3 and C4 tested as negative. Coagulation profile was normal. Echocardiography revealed moderate PAH without any structural heart abnormalities. 


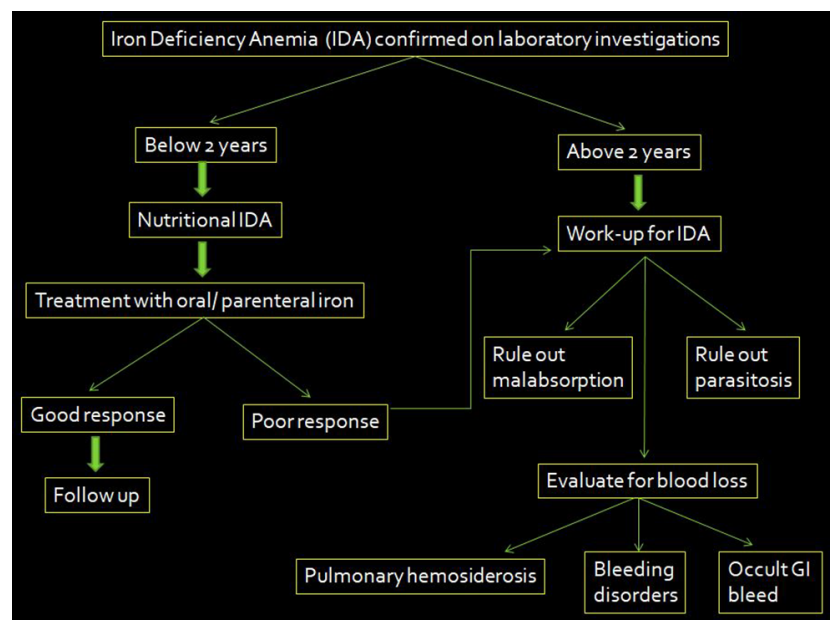

Fig. 6 Algorithm for approach to pediatric iron deficiency anemia.

Further, a bronchoscopic alveolar lavage (BAL) was performed and cytopathological analysis of BAL fluid with Perl's Prussian blue stain (-Fig. 4A) and May-Grunwald-Giemsa stain ( - Fig. 4B) depicted the classical hemosiderin-laden macrophages (siderophages), confirming the diagnosis of idiopathic pulmonary hemosiderosis (IPH). BAL fluid culture and sensitivity tested negative for tuberculosis. The child was started on oral prednisolone and currently has shown marked clinical improvement after 2 weeks of therapy.

\section{Discussion}

Pulmonary hemosiderosis is characterized by deposition and aggregation of hemosiderin in the lungs due to recurrent alveolar capillary hemorrhage ${ }^{1}$ and can be primary or secondary. Primary pulmonary hemosiderosis or IPH is a diagnosis of exclusion after ruling out secondary causes of pulmonary hemorrhage like vasculitis, autoimmune disorders, coagulation disorders, or mitral stenosis. ${ }^{2}$ IPH is a rare disease with an incidence of 0.24 to 1.23 cases/million. ${ }^{3}$ Its pathogenesis is unclear, with autoimmune, environmental, allergic, and genetic etiologies being hypothesized for the same. ${ }^{4}$ Association of IPH with celiac disease has been described, which is called as "Lane-Hamilton syndrome."

IPH is typically diagnosed within 10 years of age with around $20 \%$ being detected late in adulthood. ${ }^{4}$ The classic triad described for this entity is hemoptysis, IDA, and pulmonary opacities on chest radiograph. ${ }^{4}$ However, the triad is rarely manifested in children, and IDA is often the only presenting feature with nonspecific respiratory symptoms or occasionally the patient may be completely asymptomatic, which precludes timely recognition of the disease. ${ }^{1}$ Children most commonly present with exertional dyspnea and cough, while hemoptysis is the major complaint in adults. ${ }^{2}$ There are two phases to the disease process, depending on which clinical features vary. Alveolar hemorrhage characterizes the acute phase, and the child presents with cough, dyspnea, and hemoptysis. ${ }^{1}$ In the chronic phase, complications of pulmonary fibrosis and PAH set in, with pulmonary hypertension, cor pulmonale, cyanosis, and clubbing being the major clinical features. ${ }^{1}$
Thoracic imaging is a useful pointer to this lung pathology and sometimes a routine chest radiograph could prevent an unnecessary delay in diagnosis. Plain radiograph and HRCT of thorax efficiently detect IPH and the imaging features correspond to pulmonary hemorrhage with or without fibrosis, depending on the phase of the disease and episodic acute exacerbation $^{1}$ (- Table 1). Magnetic resonance imaging is not necessary for diagnosis, though affected lung regions have been reported to show hyperintense signal on T1-weighted and hypointense signal on T2-weighted images, respectively. ${ }^{1}$ Lymphadenopathy, hemothorax, and fibrothorax have also been described in this condition. ${ }^{1}$

However, imaging features are not pathognomonic for IPH as these may be seen in any case of diffuse alveolar hemorrhage (DAH). The radiological differential diagnosis for DAH would primarily include congenital heart disease, especially mitral stenosis and vasculitis like Goodpasture's syndrome, granulomatosis with polyangiitis, systemic lupus erythematosus, rheumatoid arthritis, celiac disease, etc. ${ }^{1}$ Gomez-Roman had classified DAH syndrome in three groups: (1) pulmonary capillaritis with positive ANCA; (2) bleeding caused by immune deposits; and (3) drug reactions, infections, and idiopathic conditions. ${ }^{3}$ Pulmonary tuberculosis is a major differential diagnosis in endemic countries. ${ }^{5}$ Other additional conditions to be ruled out would be coagulation disorders, neoplasms, and rarer disorders such as pulmonary veno-occlusive disease and pulmonary capillary hemangiomatosis. ${ }^{1}$ Exposure to toxins like cocaine, pesticides, and insecticides may also present with similar radiological picture. ${ }^{1}$ Hence, adequate workup to rule out vasculitis, collagen vascular diseases, infections, and congenital heart diseases is warranted before diagnosing IPH. A clinicoradiological approach to pulmonary hemosiderosis has been outlined in - Fig. $\mathbf{5}$.

BAL is highly specific for pulmonary hemosiderosis with the detection of siderophages being pathognomonic. ${ }^{6}$ However, lung biopsy is the gold standard as it definitively rules out other vasculitic changes. ${ }^{7}$

Table 1 Imaging features of idiopathic pulmonary hemosiderosis on chest radiograph and high-resolution computed tomography (HRCT) in acute and chronic phases of the disease

\begin{tabular}{|l|l|l|}
\hline $\begin{array}{l}\text { Chest } \\
\text { radio- } \\
\text { graph }\end{array}$ & $\begin{array}{l}\text { Airspace opacities/consoli- } \\
\text { dation in perihilar and lower } \\
\text { lobe }\end{array}$ & $\begin{array}{l}\text { Reticular opacities in } \\
\text { areas of consolidation } \\
\text { especially basilar and } \\
\text { subpleural location }\end{array}$ \\
\hline HRCT & $\begin{array}{l}\text { Ground-glass opacity (GGO) } \\
\text { and consolidation in diffuse } \\
\text { pattern or hilar, perihilar, } \\
\text { and lower lobe distribution. } \\
\text { Interstitial deposition of } \\
\text { hemosiderin laden mac- } \\
\text { rophages leading to inter- } \\
\text { lobular septal thickening, } \\
\text { which when superimposed } \\
\text { on areas of GGO gives a } \\
\text { "crazy paving" appearance }\end{array}$ & $\begin{array}{l}\text { Pulmonary fibrosis } \\
\text { and honeycombing. } \\
\text { Intra- and interlobular } \\
\text { septal thickening with } \\
\text { traction bronchiectasis } \\
\text { and bronchiolectasis. } \\
\text { Progressive massive } \\
\text { fibrosis (PMF) with high } \\
\text { attenuation consolida- } \\
\text { tion and centrilobular } \\
\text { nodules due to intra-al- } \\
\text { veolar macrophages }\end{array}$ \\
\hline \multicolumn{2}{|c}{} \\
\hline
\end{tabular}


Table 2 Laboratory findings on blood examination in iron deficiency anemia

\begin{tabular}{|l|l|}
\hline RBC count & low $\left(<4\right.$ million $\left./ \mathrm{mm}^{3}\right)$ \\
\hline Hemoglobin & $\begin{array}{l}\text { Low for age and gender }(<12 \mathrm{mg} / \mathrm{dL} \text { for } \\
\text { females, }<13 \mathrm{mg} / \mathrm{dL} \text { for males })\end{array}$ \\
\hline Hematocrit & $\begin{array}{l}\text { Low for age and gender }(<36 \% \text { for } \\
\text { females and } 41 \% \text { for males })\end{array}$ \\
\hline MCV & $<72 \mathrm{fL}$ \\
\hline MCH & $<27 \mathrm{pg}$ \\
\hline MCHC & $<30 \%$ \\
\hline Peripheral smear & $\begin{array}{l}\text { Hypochromia, microcytosis, anisochro- } \\
\text { mia, anisocytosis }\end{array}$ \\
\hline Serum ferritin & $<12 \mathrm{ng} / \mathrm{mL}$ \\
\hline Serum iron & $<30 \mu \mathrm{g} / \mathrm{dL}$ \\
\hline $\begin{array}{l}\text { Total iron binding } \\
\text { capacity }\end{array}$ & $>480 \mu \mathrm{g} / \mathrm{dL}$ \\
\hline $\begin{array}{l}\text { Transferrin } \\
\text { saturation }\end{array}$ & $<16 \%$ \\
\hline RDW & $>14$ \\
\hline
\end{tabular}

Abbreviations: $\mathrm{MCH}$, mean corpuscular hemoglobin; $\mathrm{MCHC}$, mean corpuscular hemoglobin concentration; MCV, mean corpuscular volume; RBC, red blood cell; RDW, red cell distribution width.

Systemic glucocorticoid is the drug of choice in the acute phase and has been found to limit the progression of fibrosis. ${ }^{5}$ Oral prednisolone 0.5 to $0.75 \mathrm{mg} / \mathrm{kg} /$ day is given for induction of treatment until pulmonary opacities on chest radiograph regress. ${ }^{5}$ The dose is then tapered accordingly and discontinued after around 18 to 24 months. ${ }^{5}$ Nonresponsive cases may be treated with immunosuppressive medication like azathioprine and hydroxychloroquine. ${ }^{6}$ A gluten-free diet is proposed in Lane-Hamilton syndrome. ${ }^{1}$ Trials of lung transplants have been unsuccessful due to the recurrence of bleed into the transplanted tissue. ${ }^{8}$ Overall, the prognosis is poor in children with mortality rate $>50 \%$ due to delay in diagnosis from lack of awareness of the entity and absence of classical triad. ${ }^{9}$ The most common cause of death is an acute or chronic respiratory failure due to massive alveolar hemorrhage or cor pulmonale, respectively. ${ }^{1}$

IDA is the most prevalent nutritional deficiency globally with children in developing countries bearing most of the brunt. ${ }^{10}$ It is characterized by hypochromic microcytopenic blood picture and low hemoglobin count, MCV, MCH and MCHC, serum ferritin, serum iron, and transferrin saturation (-Table 2). ${ }^{10}$ Though the most common cause of IDA is nutritional, blood loss as an underlying cause should be considered in older children, especially when there is a poor response to oral iron treatment and the requirement of blood transfusion. ${ }^{10}$ A detailed clinical history may give clue to etiologies like the passage of worms in stool in parasitosis or menorrhagia in adolescent girls with von Willebrand factor deficiency. Occult gastrointestinal bleeding and bleeding disorders have to be ruled out primarily. Occult stool blood may be positive in cases of Meckel's diverticulum, gastrointestinal polyp, peptic ulcer, inflammatory bowel disease, intestinal hemangioma or vascular malformations. Other rare but significant causes to be assessed are pulmonary hemosiderosis and malabsorption from celiac disease or chronic diarrhea. ${ }^{10} \mathrm{~A}$ simplified approach to pediatric IDA has been outlined in - Fig. 6 .

\section{Conclusion}

IPH in children often has a severe disease course with a poor prognosis due to irreversible fibrosis and PAH that sets in. ${ }^{2}$ Early diagnosis of the condition with the institution of corticosteroid therapy alone or in combination with immunosuppressants, however, has shown a promising role during acute exacerbations and maintenance therapy of $\mathrm{IPH}^{8}{ }^{8}$ Treatment of anemia is only supportive and detection of the underlying cause of blood loss is the key to accurate management of pediatric IDA. Hence, awareness of the condition, systematic approach to IDA, and high clinical suspicion with early referral for thoracic imaging is essential for prompt detection of pediatric IPH, as hemoptysis is not a consistent feature in children.

\section{Funding}

This study has not received funding/sponsorship of any sort, including study design, collection, analysis, and interpretation of data, writing of the report, decision to submit the paper for publication.

\section{Conflict of Interest}

There are no conflicts of interests, real or perceived, among authors.

\section{Acknowledgments}

None.

\section{References}

1 Khorashadi L, Wu CC, Betancourt SL, Carter BW. Idiopathic pulmonary haemosiderosis: spectrum of thoracic imaging findings in the adult patient. Clin Radiol 2015;70(5):459-465

2 Tzouvelekis A, Ntolios P, Oikonomou A, et al. Idiopathic pulmonary hemosiderosis in adults: a case report and review of the literature. Case Rep Med 2012;2012:267857

3 Kamienska E, Urasinski T, Gawlikowska-Sroka A, Glura B, Pogorzelski A. Idiopathic pulmonary hemosiderosis in a 9-year-old girl. Eur J Med Res 2009;14(Suppl 4) :112-115

4 Taytard J, Nathan N, de Blic J, et al. French RespiRare ${ }^{\circledR}$ group. New insights into pediatric idiopathic pulmonary hemosiderosis: the French RespiRare (®) cohort. Orphanet J Rare Dis 2013;8:161

5 Shamim S, Biswas S, Mandal S. Idiopathic pulmonary hemosiderosis: a differential diagnosis of pulmonary tuberculosis in a young child. Indian J Med Spec 2019;10(3):170

6 Bakalli I, Kota L, Sala D, et al. Idiopathic pulmonary hemosiderosis - a diagnostic challenge. Ital J Pediatr 2014;40(1):35

7 Bhatia S, Tullu MS, Vaideeswar P, Lahiri KR. Idiopathic pulmonary hemosiderosis: alveoli are an answer to anemia. J Postgrad Med 2011;57(1):57-60

8 Ioachimescu OC, Sieber S, Kotch A. Idiopathic pulmonary haemosiderosis revisited. Eur Respir J 2004;24(1):162-170

9 Koker SA, Gözmen S, Oymak Y, et al. Idiopathic pulmonary hemosiderosis mimicking iron deficiency anemia: a delayed diagnosis? Hematol Rep 2017;9(2):7048

10 Özdemir N. Iron deficiency anemia from diagnosis to treatment in children. Turk Pediatri Ars 2015;50(1):11-19 